\title{
ESTUDO DA MORFOLOGIA DE PELOTAS DE MINÉRIO DE FERRO REDUZIDAS POR AGENTES GASOSOS
}

\author{
Girley Ferreira Rodrigues ' \\ Eduardo Junca ${ }^{2}$ \\ Thomaz Augusto Guisard Restivo ${ }^{3}$ \\ José Roberto de Oliveira ${ }^{2}$ \\ Denise Crocce Romano Espinosa ${ }^{4}$
}

\section{Resumo}

O estudo da morfologia de pelotas é uma importante ferramenta para compreender possíveis mecanismos envolvidos na redução. Assim, este trabalho tem por objetivo caracterizar três diferentes tipos de minérios e correlacionar a morfologia obtida na redução de pelotas fabricadas a partir destes minérios. A redução foi realizada em um forno tubular horizontal em $850^{\circ} \mathrm{C}$ em duas atmosferas redutoras. A primeira contendo hidrogênio e a segunda contendo hidrogênio + monóxido de carbono. Análise granulométrica, área superficial e difração de raios-X foram realizadas para a caracterização dos minérios. Microscópio eletrônico de varredura foi utilizado no estudo da morfologia das pelotas reduzidas. Os resultados mostraram que a diminuição da distribuição granulométrica do minério de pellet feed ocasiona um maior número de whisker. Além disso, foi observado que a presença de monóxido de carbono na mistura redutora proporcionou um menor número de poros e uma maior formação de whisker.

Palavras-chave: Caracterização mineralógica; Caracterização morfológica; Pellet feed; Minério de ferro.

\section{STUDY OF IRON ORE PELLETS MORPHOLOGY REDUCED BY GASEOUS AGENTS}

\begin{abstract}
The study of pellets morphology is an important tool to understand possible mechanisms involved during its reduction reaction. Thus, the aim of this paper is characterize three different iron ores and correlate its morphology obtained after the complete reduction with its features. The reductions tests were accomplished in a horizontal tubular furnace at $850^{\circ} \mathrm{C}$. Two reducing atmosphere were used. The first one contained hydrogen. The second one was a mixture between first gas + carbon monoxide. The iron ore characterization was carried out by size analysis, surface area and X-ray analysis. Scanning electron microscopy was used to obtain the morphology from the reduced pellets. The results showed a greater number of whisker were obtained with more fine ore. Furthermore, it was noted that carbon monoxide provided a lesser number of pores and greater number of whisker.
\end{abstract}

Keywords: Mineralogical characterization; Morphological characterization; Pellet feed; Iron ore.

\section{INTRODUÇÃO}

Durante a reação de redução de óxidos de ferro, diversas morfologias podem ser obtidas. Condições experimentais, como por exemplo, temperatura e composição do gás redutor, afetam diretamente a redução e, consequentemente, a morfologia dos produtos obtidos. Desta forma, ferro com camadas porosas [1-5] ou densas $[3,4,6]$ podem ser obtidas.
Importantes estudos sobre morfologias do ferro obtido durante a redução tem sido realizado por $\mathrm{St}$ John et al. [3,4,7] em uma série de publicações. Os autores obtiveram três diferentes tipos de microestruturas.

O primeiro tipo corresponde a formação de um ferro poroso após a completa redução da wustita. $O$ segundo tipo é caracterizado pela formação de ferro poroso na wustita

'Universidade de Mogi das Cruzes - UMC, São Paulo, SP, Brasil. E-mail: girleyf@gmail.com

${ }^{2}$ Instituto Federal de Educação, Ciência e Tecnologia do Espírito Santo - IFES, Vitória, ES, Brasil.

${ }^{3}$ Universidade de Sorocaba - UNISO, Sorocaba, SP, Brasil.

${ }^{4}$ Departamento de Engenharia Química, Universidade de São Paulo - USP, São Paulo, SP, Brasil. 
densa. Por fim, na terceira microestrutura não é observada a presença de poros.

Além das morfologias citadas anteriormente, alguns autores [4,6,8-10] têm observado a formação de nódulos de ferro em forma de saliências alongadas na camada de ferro obtida após a redução, na qual denominou-se whiskers. Estes crescem para fora da camada de ferro.

O aparecimento desta fase é conhecido como sendo um dos responsáveis pelo inchamento catastrófico que ocorrem em pelotas de minério de ferro ou de óxidos de ferro durante a redução.

Pesquisas tem mostrado que o crescimento de whisker é favorecido por óxidos básicos, como por exemplo, $\mathrm{CaO}$, $\mathrm{Na}_{2} \mathrm{O}$ e $\mathrm{K}_{2} \mathrm{O}[8, \mathrm{II}]$.

Alguns pesquisadores $[9, \mathrm{II}]$ tem mencionado o mecanismo sugerido por Wagner como o início do processo de formação do whisker. De acordo com esta teoria, na redução de wustita para ferro metálico, os íons de oxigênio ligados à wustita são transportados por difusão pelo o gás redutor, gerando vacâncias no reticulado. Estas vacâncias migram do reticulado de ferro para a superfície da wustita. Além disso, ocorre um aumento da proporção $\mathrm{Fe} / \mathrm{O}$ da wustita na superfície, gerando um fluxo em direção a superfície da wustita. $O$ aumento de ferro na superfície ocasiona um aumento da atividade de ferro na superfície da wustita, que neste momento, é maior do que a atividade de ferro no reticulado da wustita. Em um determinado momento da redução, a atividade atinge um valor crítico, que neste momento é suficiente para iniciar a formação do núcleo de ferro. No núcleo de ferro, a atividade do mesmo é unitária, o que pode causar um novo gradiente da wustita supersaturada de ferro para o núcleo, o que vem a causar o crescimento do núcleo.

Desta forma, o objetivo deste trabalho é realizar a caracterização de três tipos de minérios e estudar a morfologia obtida na redução direta de pelotas fabricadas a partir destes minérios, com atmosferas contendo hidrogênio e uma mistura de hidrogênio e monóxido de carbono.

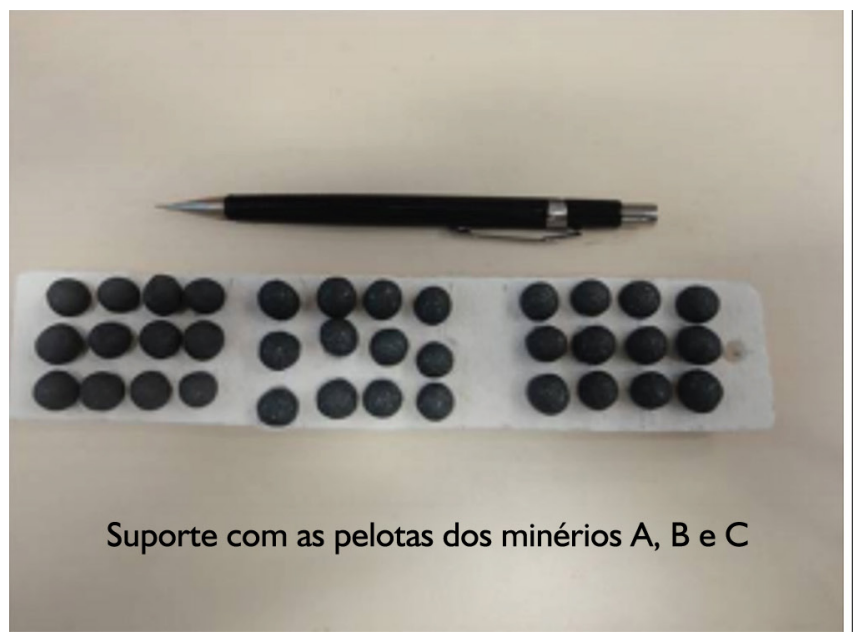

\section{MATERIAIS E MÉTODOS}

Foram utilizados três tipos de minérios de ferro, denominados Pellets feeds A, B e C.

Inicialmente, os minérios foram caracterizados por análise granulométrica por difração a laser, área superficial por BET e difração de raios-X. Em adição, foi calculada a porcentagem de cada fase obtida na difração de raios- $X$ pelo método Rietveld.

Após a caracterização dos três tipos de minérios, pelotas foram confeccionadas a partir de cada minério (denominadas como pelotas A, B e C). Esta etapa foi desenvolvida com auxílio de um disco pelotizador adaptado em um betoneira. Para obtenção de um lote homogêneo de pelotas, as mesmas foram classificadas através de peneiras de 12 e $9,52 \mathrm{~mm}$, onde apenas as pelotas entre estas peneiras foram utilizadas.

Com o intuito de investigar a morfologia das pelotas durante o processo de redução, foram realizados testes de redução completa das pelotas $A, B$ e $C$ em um forno elétrico tubular horizontal. Os ensaios foram realizados na temperatura de $850^{\circ} \mathrm{C}$.

Duas misturas redutoras foram utilizadas. A primeira trata-se de uma mistura pré-preparada contendo $90 \%$ de argônio + $10 \%$ de hidrogênio, denominada Ar- $90 \% / \mathrm{H}_{2}-10 \%$. Foi escolhida esta mistura por se tratar de uma mistura não explosiva. A segunda mistura foi confeccionada adicionando-se $\mathrm{CO}$ à primeira mistura, respeitando a proporção de $75 \%$ de hidrogênio e $25 \%$ de CO, conforme Equação I [ I 2, I3]. Tal mistura foi denominada $\mathrm{H}_{2}-75 \% / C O-25 \%$.

$$
\mathrm{CH}_{4}+\mathrm{H}_{2} \mathrm{O}=3 \mathrm{H}_{2}+\mathrm{CO}
$$

As pelotas $A, B$ e $C$ foram colocadas em um suporte refratário de alumina que foi inserido no forno horizontal (Figura I). Foi realizado o aquecimento das amostras dentro do forno até a temperatura de $850^{\circ} \mathrm{C}$ sob o fluxo de nitrogênio. Atingida e estabelecida a temperatura, o fluxo de gás inerte

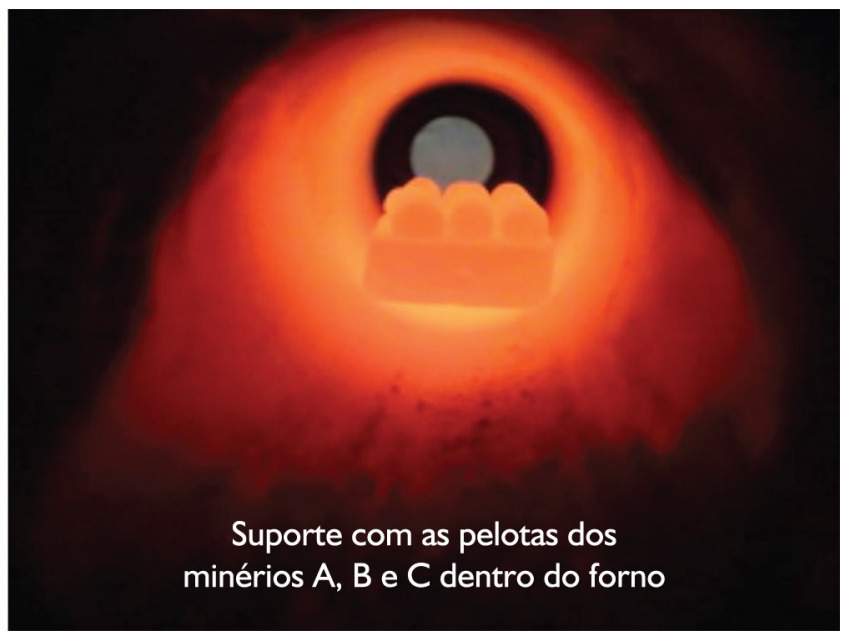

Figura I. Suporte refratário de alumina para alocação das pelotas A, B e C. 
foi substituído pelo fluxo de gás redutor. As pelotas foram retiradas do forno em tempos espaçados de I5, 30, 45, 60, $90,120,150,210,270,330,390$ e 450 minutos e resfriadas sob atmosfera de nitrogênio para evitar a reoxidação.

As pelotas $A, B$ e $C$ foram ensaiadas ao mesmo tempo. Foi retirada em cada tempo uma pelota de cada um dos três tipos de pellets feeds.

Utilizou-se um forno elétrico, marca Lindberg Blue, com temperatura de trabalho até $1.200^{\circ} \mathrm{C}$. O forno horizontal foi conectado a um controlador de temperatura Lindberg Blue. A tensão, frequência e potência utilizadas pelo forno foram $208 / 240 \mathrm{~V}, 50 / 60 \mathrm{~Hz}$ e $5000 \mathrm{~W}$, respectivamente. Duas flanges de aço inoxidável vedavam as extremidades do tubo de mulita. O controle do fluxo dos gases de entrada foi feito utilizando um controlador de fluxo mássico da MKS modelo 647B com 4 canais.

Após a completa redução, as pelotas foram encaminhadas para análise no microscópio eletrônico de varredura para realizar a análise da morfologia das pelotas. Foi necessário o recobrimento das mesmas com ouro para obtenção das imagens no microscópio.

\section{RESULTADOS E DISCUSSÃO}

\section{I Caracterização dos Pellets Feeds}

A Tabela I mostra a composição das fases presentes nos pellets feeds $A, B$ e $C$ obtidos pelo método Rietveld de quantificação de fases.

Os três pellets feeds apresentaram a hematita como principal fase. Entretanto, foi observado que somente no pellet feed $\mathrm{C}$ foram detectadas impurezas, neste caso, foi identificada na forma de quartzo.
A Tabela 2 mostra os resultados das análises granulométricas das três amostras de pellets feeds.

Os parâmetros DI0, D50 e D90 indicam que $10 \%$, $50 \%$ e $90 \%$ das partículas, respectivamente, estão abaixo do valor indicado. Desta forma, nota-se que a amostra do pellet feed $C$ apresenta a menor distribuição granulométrica, onde $90 \%$ das partículas são menores que $54,50 \mu \mathrm{m}$, enquanto que a amostra de pellet feed $\mathrm{A}$ apresentou a maior distribuição granulométrica, com $90 \%$ das partículas menores que $122,79 \mu \mathrm{m}$.

A Tabela 3 apresenta os resultados obtidos análise da área superficial das três amostras de pellets feeds.

Observa-se que a amostra de pellet feed $\mathrm{C}$ apresentou maior área superficial em relação as outras amostras. Estes valores condizem com os valores obtidos na análise granulométrica, uma vez que quanto menor a granulometria de uma determinada amostra, maior é sua área superficial.

\subsection{Caracterização Morfológica das Pelotas Reduzidas}

As Figuras 2 e 3 mostram, respectivamente, imagens de elétrons secundários obtidos via microscópio eletrônico de varredura das pelotas produzidas pelos três pellets feeds reduzidas pelas misturas $\mathrm{Ar}-90 \% / \mathrm{H}_{2}-10 \%$ e $\mathrm{H}_{2}-75 \% / \mathrm{CO}-25 \%$.

Visualmente, observou-se uma maior formação de whiskers na pelota $C$ do que nas pelotas $A$ e $B$. Este fato pode estar relacionado com as impurezas contidas no pellet feed C. De acordo com Chang e De Jonghe [I I], a presença de $\mathrm{SiO}_{2}$ não promove a formação de whiskers, entretanto, esta fase aumenta a taxa de nucleação de metal, que pode estar associada a formação dos mesmos. Além disso, Nicolle e Rist [9] citam que partículas menores favorecem o crescimento de whiskers. Conforme foi observado na

Tabela I. Quantificação das fases presentes nos pellets feeds A, B e C pelo método Rietveld

\begin{tabular}{cccc}
\hline Fases & $\begin{array}{c}\text { Pellet feed A } \\
\text { (\% em peso) }\end{array}$ & $\begin{array}{c}\text { Pellet feed B } \\
\text { (\% em peso) }\end{array}$ & $\begin{array}{c}\text { Pellet feed C } \\
\text { (\% em peso) }\end{array}$ \\
\hline Hematita & 100 & 100 & 88,5 \\
Magnetita & - & - & 6,2 \\
Goethita & - & - & 4,2 \\
Quartzo & - & - & 1,1 \\
\hline
\end{tabular}

Tabela 2. Análise granulométrica dos pellets feeds A, B e C obtidos por difração a laser

\begin{tabular}{cccc}
\hline Parâmetros & $\begin{array}{c}\text { Pellet feed A } \\
(\mu \mathrm{m})\end{array}$ & $\begin{array}{c}\text { Pellet feed B } \\
(\mu \mathrm{m})\end{array}$ & $\begin{array}{c}\text { Pellet feed C } \\
(\mu \mathrm{m})\end{array}$ \\
\hline D10 & 19,46 & 18,88 & 1,90 \\
D50 & 47,64 & 41,80 & 19,79 \\
D90 & 122,79 & 107,81 & 54,50 \\
\hline
\end{tabular}

Tabela 3. Área superficial dos pellets feeds A, B e C obtidos por análise de BET

\begin{tabular}{cccc}
\hline Amostras & Pellet feed A & Pellet feed B & Pellet feed C \\
\hline Área superficial $\left(\mathrm{m}^{2} / \mathrm{g}\right)$ & 2,16 & 2,23 & 15,29 \\
\hline
\end{tabular}




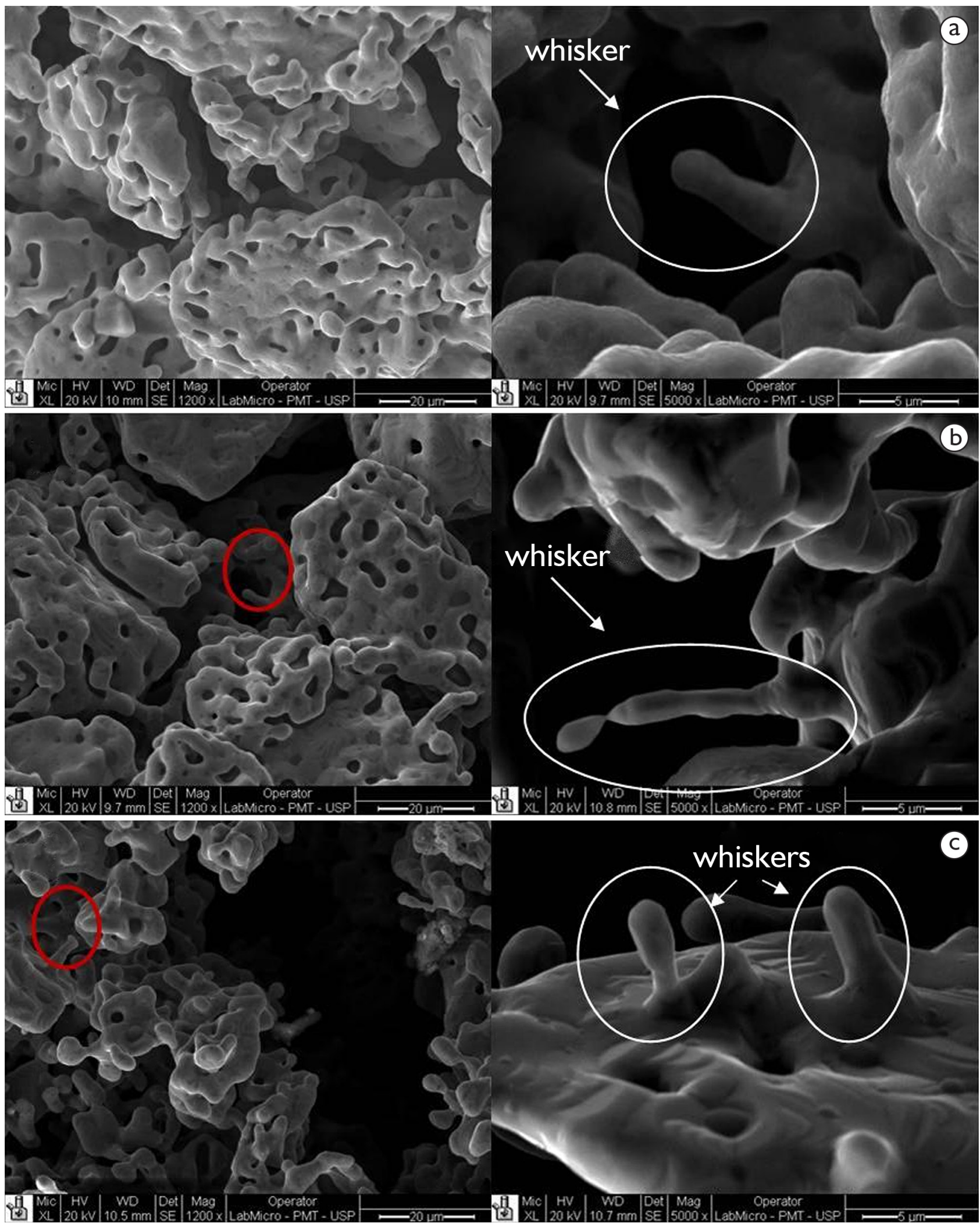

Figura 2. Imagens de elétrons secundários obtidas pelo microscópio eletrônico de varredura da superfície das pelotas totalmente reduzidas via mistura Ar-90\%/ $\mathrm{H}_{2}-10 \%$. (a) pelota $\mathrm{A}$, (b) pelota B e (c) pelota $\mathrm{C}$. 


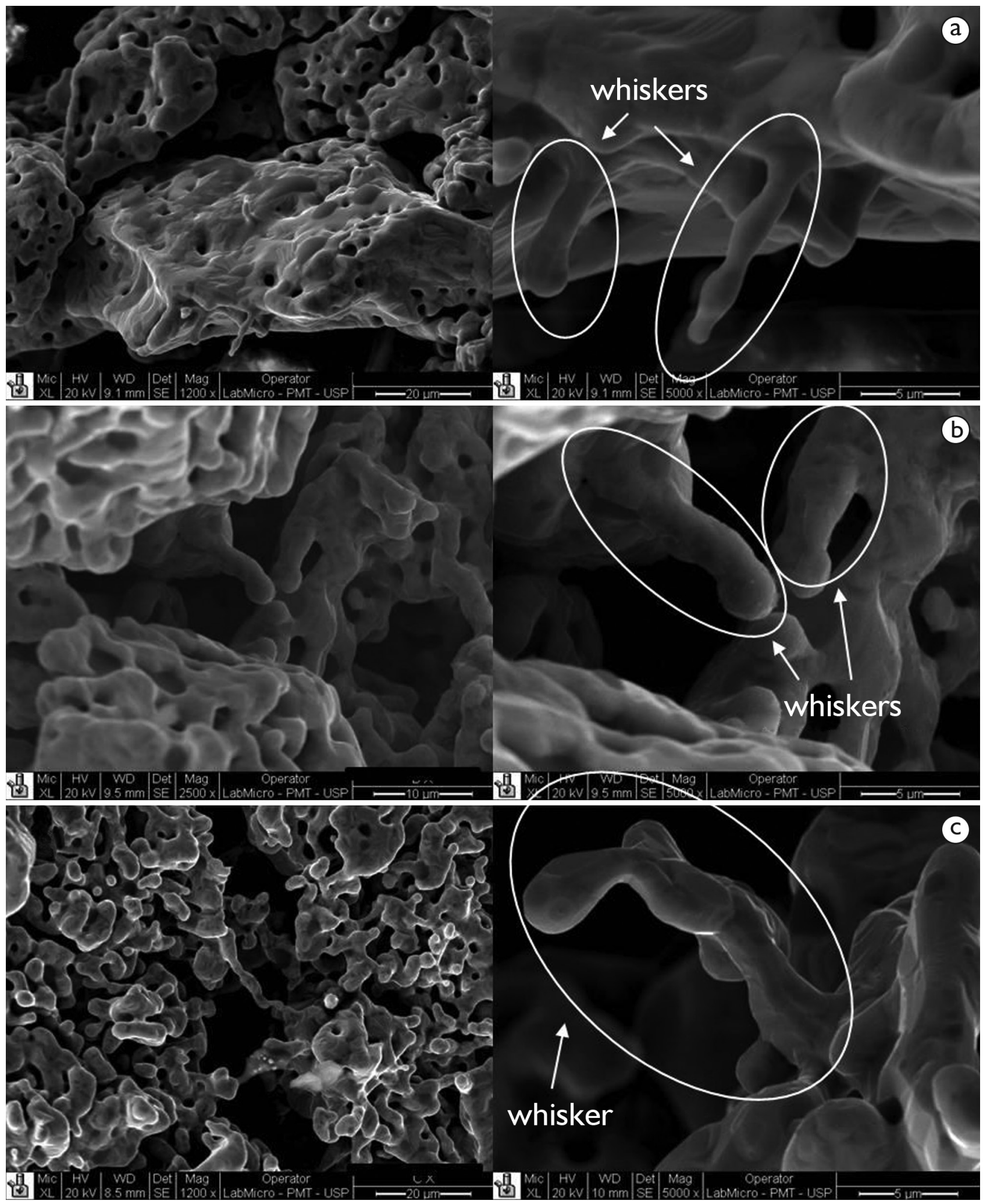

Figura 3. Imagens de elétrons secundários obtidas pelo microscópio eletrônico de varredura da superfície das pelotas totalmente reduzidas via mistura $\mathrm{H}_{2}-75 \% / C O-25 \%$. (a) pelota $\mathrm{A}$, (b) pelota B e (c) pelota C. 
análise granulométrica, o pellet feed $\mathrm{C}$ apresentou uma menor distribuição granulométrica, o que pode favorecer a formação do whisker.

Utilizando-se a mistura Ar- $90 \% / \mathrm{H}_{2}-10 \%$, foi observado visualmente que o ferro obtido apresentou um maior volume de poros do que o ferro obtido na redução utilizando a mistura $\mathrm{H}_{2}-75 \% / \mathrm{CO}-25 \%$. Além disso, whiskers apareceram em maior número nas pelotas reduzidas pela mistura $\mathrm{H}_{2}-75 \% / C O-25 \%$. Esta afirmação também é mencionada por Yi et al. [14]. Em adição, observações indicam que o gás de redução exerce influência na morfologia final das pelotas. O CO presente na mistura $\mathrm{H}_{2}-75 \% / \mathrm{CO}-25 \%$ aparentemente ocasionou a formação de um maior número de whiskers. Além disso, estes são maiores do que os formados pela mistura Ar-90\%/ $\mathrm{H}_{2}-10 \%$. Tal fato também é descrito por outros autores $[15,16]$. No caso da mistura $\mathrm{Ar}-90 \% / \mathrm{H}_{2}-10 \%$, o teor de $\mathrm{H}_{2}$ na mistura é maior do que o teor presente na mistura $\mathrm{H}_{2}-75 \% / \mathrm{CO}-25 \%$. $\mathrm{O} \mathrm{H}_{2}$ apresenta maior poder de reação do que o $\mathrm{CO}$, o que leva a formação de um maior número de sítios ativos para o início da nucleação do ferro reduzido. Os sítios combinam-se entre si levando a formação de uma camada porosa de ferro reduzido.

\section{CONCLUSÕES}

A partir dos resultados obtidos neste trabalho, pode-se concluir que com a diminuição da distribuição granulométrica do pellet feed, um maior número de whiskers foi obtido. Além disso, foi observado que a introdução de monóxido de carbono na mistura redutora proporcionou um menor número de poros e uma maior formação de whiskers, uma vez que a introdução de monóxido de carbono diminui a reatividade do gás redutor, e consequentemente, um menor número de sítios ativos são formados, o que favorece a formação de ferro poroso.

\section{Agradecimentos}

Os autores agradecem à Universidade de São Paulo, ao Instituto Federal de Educação, Ciência e Tecnologia do Espírito Santo, à Coordenação de Aperfeiçoamento de Pessoal de Nível Superior (Capes) e à Fundação de Amparo à Pesquisa e Inovação do Espírito Santo (FAPES).

\section{REFERÊNCIAS}

I Turkdogan ET, Vinters JV. Gaseous reduction of iron oxides: Part I. Reduction of hematite in hydrogen. Metallurgical Transactions. 197I;2(I I):3 I 75-3 I88. http://dx.doi.org/I0.1007/BF028I4970.

2 El-Geassy AA, Rajakumar V. Influence of particle size on the gaseous reduction of wustite at $900-1100^{\circ} \mathrm{C}$. Transactions of the Iron and Steel Institute of Japan. 1985;25(I 2): I 202-I 2 I I. http://dx.doi.org/ I0.2355/ isijinternational I 966.25. I 202.

3 St John DH, Hayes PC. Microstructural features produced by the reduction of wustite in hydrogen/water gas mixtures. Metallurgical Transactions. B, Process Metallurgy. 1982; I 3B(I): I I 7- I 24. http://dx.doi.org/I0.1007/ BF02666962.

4 St John DH, Mathews SP, Hayes PC. Establishment of product morphology during the initial stage of wustite reduction. Metallurgical Transactions. B, Process Metallurgy. 1984; I5(4):709-7I7. http://dx.doi.org/I0.1007/ BF02657293.

5 Turkdogan ET, Vinters JV. Gaseous reduction of iron oxides: Parte III. Reduction-oxidation of porous and dense iron oxides and iron. Metallurgical Transactions. 1972;3(6): I 56 I-I570. http://dx.doi.org/ I 0. I007/BF02643047.

6 Gaballah I, Bert P, Dufour LC, Gleitzer C. Kinetics of the reduction of wustite by hydrogen and carbon monoxide + hydrogen mixtures. Les Memoires Scientifiques de la Revue de Metallurgie. 1972;69:523-530.

7 St John DH, Mathew SP, Hayes PC. The breakdown of dense iron layers on wustite in $\mathrm{CO} / \mathrm{CO} 2$ and $\mathrm{H} 2 / \mathrm{H} 2 \mathrm{O}$ systems. Metallurgical Transactions. B, Process Metallurgy. 1984; I5(4):70I-708. http://dx.doi.org/I0.1007/ BF02657292.

8 Gransden JF, Sheasby JS. Sticking of iron-ore during reduction by hydrogen in a fluidized-bed. Canadian Metallurgical Quarterly. 1974;3(4):649-657. http://dx.doi.org/10.1 179/cmq.1974.13.4.649.

9 Nicolle R, Rist A. The mechanism of whisker growth in the reduction of wustite. Metallurgical Transactions. B, Process Metallurgy. 1979; I0B(3):429-438. http://dx.doi.org/10.1007/BF026525 I6.

IO El Moujahid S, Rist A. The nucleation of iron on dense wustite: a morphological study. Metallurgical Transactions. B, Process Metallurgy. 1988; I9B(5):787-802. http://dx.doi.org/I0. 1007/BF02650198.

I I Chang M, De Jonghe LC. Whisker growth in reduction of oxides. Metallurgical Transactions. B, Process Metallurgy. I984; I5B(4):685-694. http://dx.doi.org/I0.1007/BF02657290. 
12 Zhang N, Lior N. Two novel oxy-fuel power cycles integrated with natural gas reforming and CO2 capture. Energy. 2008;33(2):340-35I. http://dx.doi.org/I0.1016/j.energy.2007.09.006.

13 Olivieri A, Vegliò F. Process simulation of natural gas steam reforming: fuel distribution optimisation in the furnace. Fuel Processing Technology. 2008;89(6):622-632. http://dx.doi.org/I0.1016/j.fuproc.2007.12.00I.

14 Yi L, Huang Z, Jiang T. Sticking of iron ore pellets during reduction with hydrogen and carbon monoxide mixtures: behavior and mechanism. Powder Technology. 2012;235:100 I-I007. http://dx.doi.org/l0.1016/j. powtec.2012.II.043.

15 Wang $\mathrm{H}$, Sohn HY. Effects of reducing gas on swelling and iron whisker formation during the reduction of iron oxide compact. Steel Research International. 20I2;83(9):903-907. http://dx.doi.org/I0. I002/srin.20I 200054.

16 Komatina M, Gudenau $\mathrm{H}$. The sticking problem during direct reduction of fine iron ore in the fluidized bed. Association of Metallurgical Engineers Serbia and Montenegro. 2004;309-328.

Recebido em: 23 Mar. 2015

Aceito em: 4 Ago. 2015 